\title{
Aerogel-based materials for adsorbent applications in material domains
}

\author{
Nur Afifah Zubair ${ }^{1,2}$,Ebrahim Abouzari-Lotf ${ }^{2,3}$, Mohamed Mahmoud Nasef ${ }^{4}$, and Ezzat \\ Chan Abdullah ${ }^{1}$ \\ ${ }^{1}$ Malaysia-Japan International Institute of Technology, Universiti Teknologi Malaysia, Jalan Sultan \\ Yahya Petra, 54100 Kuala Lumpur, Malaysia. \\ ${ }^{2}$ Center of Hydrogen Energy, Institute of Future Energy, Universiti Teknologi Malaysia, Jalan Sultan \\ Yahya Petra, 54100 Kuala Lumpur, Malaysia. \\ ${ }^{3}$ School of Chemical and Energy Engineering, Faculty of Engineering, Universiti Teknologi Malaysia, \\ 81310 Johor Bahru, Johor, Malaysia. \\ ${ }^{4}$ Department of Chemical Engineering, Faculty of Engineering, Universiti Teknologi PETRONAS, \\ Bandar Seri Iskandar, 31750 Tronoh, Perak, Malaysia.
}

\begin{abstract}
Aerogels are considered to be promising materials in various applications due to their exclusive properties. Over the last decades, the potential of organic, inorganic, or hybrid aerogels has been practically exploited in different fields of use. Some aerogel compositions have been patented recently but their application in the area of adsorption remains limited. This review intends to discuss the potential of aerogels as adsorbents, which is summarised from the more recent progressive research and their capabilities. Furthermore, the potential of aerogels as viable absorbents for environmental remediation is also discussed. After a short introduction covering the aerogel properties, preparation procedures, and their possible classification options, the review is structured based on their possible use as adsorbents.
\end{abstract}

\section{Introduction}

Aerogels are highly porous light materials possessing a number of exceptional and unique physical properties, which attract the attention of researchers in various areas of science and technology. Most of their noticeable characteristics enable fine-tuning of aerogels, therefore lead to highly tailorable final products [1,2]. Aerogels exhibit abundant of excellent properties including low thermal conductivity, transparency, flexibility, extremely high porosity, low weight and density, large surface area, high mechanical strength, and ultralow dielectric constant, which make them uniquely suitable for a high range of applications in very different fields [3-5]. Steven Kistler prepared the first nanoporous ultralight aerogels in 1931 [1]. Recently, the interest of aerogels is growing, as indicated by the number of publications each year [6-10]. Based on the numerous findings in relation to aerogel properties, the material has attracted a tremendous attention of many 
researchers. Their advantages over other similar materials and the possibility of turning natural resources into aerogels have led to high interest for further investigation.

Aerogels can be obtained in various sizes and shapes, ranging from beads to disks to monoliths, by the removal of the liquid from a corresponding gel $[11,12]$. The liquid phase is replaced with air using either freeze-drying or supercritical carbon dioxide $\left(\mathrm{CO}_{2}\right)$ drying techniques. The latter preserves the original structure of the gel, which leads to the creation of large surface areas. Aerogels are most commonly synthesised by using a sol-gel procedure-based initial preparation step, followed by drying using supercritical $\mathrm{CO}_{2}[13$, 14]. Some of the most studied aerogel-based applications include Cherenkov counters [1517], different aerospace-related applications [18, 19], thermal superinsulation [20], and others [21, 22]. Recently, an increasing interest in aerogels can be observed in pharmaceutical sciences [23], food-related technologies [24], biosensors, [25] and biotechnology [26].

\section{Classification}

Aerogels can be classified according to their appearance as monoliths, powders, or films in different types of structure such as microporous, mesoporous, and mixed porous. The most often used classification is based on their composition and in general, describes three aerogel categories: inorganic, organic, and inorganic-organic hybrid aerogels. The broadest range of various aerogels can be found in the category of inorganic aerogels. These aerogels are prepared from alkoxides and include aerogels from various metal oxides, e.g., silicon [27], alumina $\left(\mathrm{Al}_{2} \mathrm{O}_{3}\right)$, titania $\left(\mathrm{TiO}_{2}\right)$, zirconia $\left(\mathrm{ZrO}_{2}\right)$, and other oxides. Silica aerogels are known as the most studied aerogel materials in inorganic chemistry. Silica aerogels can possess exceptional properties, which are the result of their nanoporous structure (porosities of 90\%-99.8\%), low density $\left(\sim 0.003 \mathrm{~g} \mathrm{~cm}^{3}\right)$, and high specific surface area (500-1200 $\mathrm{m}^{2} \mathrm{~g}^{-1}$ [4]. Typically, silica aerogels exhibit pores in the range of 5-100 nm and an average pore diameter between 20 and $40 \mathrm{~nm}$. A very important related characteristic of silica aerogels is their mesoporosity that can be controlled through tailoring of the sol-gel process conditions. Silica aerogels are among the best-known thermal (thermal conductivity $\sim 3 \mathrm{~mW} \mathrm{~m} \mathrm{~K}^{-1}$, measured at ambient temperature and pressure) and acoustic insulating materials (acoustic velocity $\sim 100 \mathrm{~m} \mathrm{~s}^{-1}$ ) [28]. They can also be made optically transparent but are also often very brittle, thus limiting their applicability in some areas. All mentioned properties make silica aerogels suitable for numerous applications, especially in thermal insulation (acting as superinsulators) for maintaining temperatures in a wide temperature range (from -78 to $\sim 650^{\circ} \mathrm{C}$ ). Different functional silica aerogels can also be found as part of clothes. Such textiles are successfully applied in clothing for people working at low temperatures (e.g., cold storage rooms in food industry), in clothing for diving, as part of aeronautical and aerospace gear, and also in firefighter suits, which have to be resistant to very high temperatures [29].

The most extensively studied organic aerogels are the resorcinol-formaldehyde (RF) and melamine-formaldehyde aerogels. Organic aerogels have some common properties despite the structural differences originating from different precursors used during preparation. Specifically, they have pores with small diameters, high specific surface areas (400-1000 $\left.\mathrm{m}^{-} \mathrm{g}^{-1}\right)$, and are present as solid matrices, which are formed from either connected colloidal particles or polymeric chains of about $10 \mathrm{~nm}$ in diameter. RF aerogels are stiffer and stronger than silica aerogels and also act as thermal superinsulators [30, 31]. 
Carbon aerogels are mostly obtained by pyrolysis, at temperatures above $500{ }^{\circ} \mathrm{C}$, of organic aerogels. In this approach, the organic aerogels transform to an electricallyconductive carbon network. During pyrolysis, the carbon aerogels obtained from RF aerogels retain high specific surface area $\left(400-800 \mathrm{~m}^{2} \mathrm{~g}^{-1}\right)$, large specific mesopore volume $\left(>0.55 \mathrm{~cm}^{3} \mathrm{~g}^{-1}\right)$, and the isotherms with a hysteresis loop of their parent organic aerogel [16]. Until recently, carbon aerogels did not exhibit fractal structures. However, a new family of carbon aerogels has been produced, which shows mass fractality with a mass fractal exponent of 2.5 according to Barbieri et al. This result was obtained with RF gelation in acetone and catalysed by perchloric acid, instead of water and sodium carbonate. Moreover, this kind of carbon aerogel displays a bimodal distribution of pores, with microand macropores, contrary to all other types of carbon aerogels described in the literature, which are mesoporous [32].

The synergistic effects where organic and inorganic aerogels are synergistically combined are derived as a hybrid aerogel material that could offer some excellent characteristics. One of the novel hybrid aerogels, syndiotactic polystyrene (sPS) aerogels, have also received considerable attention since it was first reported by Daniel et al. in 2005 . In contrast to silica aerogels, the intermolecular physical bonding of the chains in the crystalline junctions holds together the three-dimensional networks in sPS aerogels. sPS aerogels offer a combination of macropores (diameter $>50 \mathrm{~nm}$ ) formed between the adjoining strands of sPS and micropores (diameter $<2 \mathrm{~nm}$ ) formed inside the individual crystalline strands. sPS aerogels show fast sorption kinetics and can absorb and imprison guest molecules, such as volatile organic compounds, into crystalline nanocavities with an apparent increase in guest molecule diffusivity of up to seven orders of magnitude with respect to sPS films. sPS aerogels do not shatter easily like inorganic silica aerogels but undergo irreversible network strand buckling under small stress. The study showed that the hybrid aerogels performed much better in terms of mechanical performance, surface area, and absorption capacity of a hydrocarbon liquid [33].

\section{Synthesis}

Silica aerogels are synthesised in the first step by traditional low-temperature sol-gel chemistry, but in contrast to xerogels where wet gels are often dried by evaporation, aerogels are essentially dried often by supercritical drying. As a result, the dried samples retain the porous texture of the wet stage. The synthesis of (silica) aerogels can be divided into three general steps: gel preparation by sol-gel processes, ageing of the gel in its mother solution to prevent the gel from shrinking during drying, and drying of the gel under special conditions to prevent the gel structure from collapsing. A simplified reaction for silica aerogels, i.e., the most common type of aerogels for insulation purposes, can be presented as follows:

$$
\mathrm{Si}\left(\mathrm{OCH}_{3}\right)_{4}+2 \mathrm{H}_{2} \mathrm{O} \leftrightarrow \mathrm{SiO}_{2}+4 \mathrm{CH}_{3} \mathrm{OH}
$$

A detailed comprehensive review on the synthesis of silica aerogels has been written recently by Dorcheh and Abbasi [34] and this work is referred for a more extensive analysis of aerogel synthesis and recent developments. 


\subsection{Gel preparation}

Sol-gel process is a process in which solid nanoparticles dispersed in a liquid agglomerate together to form a continuous three-dimensional network extending throughout the liquid. The process is extensively described by Brinker and Scherer [35]. Aerogels are essentially the solid framework of such a gel isolated from its liquid medium. For silica aerogels, nanoparticles are grown directly in a liquid.

The main precursors for silica aerogels are silicon alkoxides. Tetramethoxysilane $\left(\mathrm{Si}\left(\mathrm{OCH}_{3}\right)_{4}\right.$ or $\left.\mathrm{TMOS}\right)$, tetraethoxysilane $\left(\mathrm{Si}\left(\mathrm{OC}_{2} \mathrm{H}_{5}\right)_{4}\right.$ or $\left.\mathrm{TEO}\right)$, and polyethoxydisiloxane $\left(\mathrm{SiO}_{\mathrm{n}}\left(\mathrm{OC}_{2} \mathrm{H}_{5}\right)_{4-2 \mathrm{n}}\right.$ or PEDS-Px) are most often used, where PEDS-Px can be obtained by reacting TEOS with a substoichiometric quantity of water in an acidic alcoholic medium. From the point of view for the application in thermal insulation, the thermal conductivities of PEDS and TMOS aerogel monoliths will be found lower compared to TEOS aerogel monoliths, whereas high-quality transparent aerogels have been developed based on TEOS. Additional solvents such as ethanol are needed and too much water will yield low-porosity gels.

Hydrolysis of silicon alkoxides is performed in the presence of a catalyst, i.e., mostly acid or base catalysis, or a two-step catalysis. The sol becomes a gel when the solid nanoparticles dispersed in it stick together to form a network of particles spanning the liquid [36]. This requires that the solid nanoparticles in the liquid collide with each other and stick together. This is easy for some nanoparticles since they contain reactive surface groups that make them stick together after colliding by bonding or electrostatic forces, whereas some nanoparticles may require the addition of an additive to make them stick together after colliding by bonding or electrostatic forces. In general, acid hydrolysis and condensation result in linear- or weakly-branched chains and microporous structures in silica sols and the resulting gelation times are generally long [4]. On the opposite, uniform particles are easily formed in base catalysis (i.e., $\mathrm{NH}_{4} \mathrm{OH}$ ) and leads to a broader distribution of larger pores [37].

\subsection{Ageing}

The moment a sol reaches the gel point, the polymerising silica species span the container containing the sol. However, the silica spine of the gel still contains a significant number of unreacted alkoxide groups. Hydrolysis and condensation may continue and sufficient time must be given for the strengthening of the silica network, which can be enhanced by controlling the $\mathrm{pH}$, concentration, and water content of the covering solution $[38,39]$.

During ageing, two different mechanisms might affect the structure of the gel: transport of the material to the neck region and dissolution of small particles into larger ones. Common ageing procedures typically involve ethanol-siloxane mixtures [40], thus adding new monomers to the solid silica network and increasing the degree of crosslinking. The result is higher stiffness and strength of the resulting gel. This ageing is diffusion controlled; transport of the material is unaffected by convection or mixing due to 
the solid silica network, whereas diffusion itself is affected by the thickness of the gel. As a result, the time required for each processing step increases as the thickness of the gel increases, thus limits the practical production of aerogels. After ageing the gel, all water still within the pores must be removed before drying, which can be easily achieved by washing the gel with ethanol and heptane. Any water left in the gel will not be removed by supercritical drying and will lead to an opaque and very dense aerogel.

\subsection{Drying}

Drying of the gel is the final and most critical step in the production process of aerogels. Drying is ruled by capillary pressure (except for supercritical drying and freezedrying), causing consequent shrinking and possible fracture due to the small pore sizes and the resulting capillary tension. As a result, two different drying processes are most often used: ambient pressure drying (APD), where capillary tension cannot be avoided, and supercritical drying (SCD), where the pore liquid is removed above the critical temperature $\left(T_{\mathrm{cr}}\right)$ and pressure $\left(P_{\mathrm{cr}}\right)$ to avoid capillary tension.

\subsubsection{Supercritical drying}

SCD is the first and most commonly used method for silica aerogels. Two different SCD methods can be distinguished: high-temperature supercritical drying (HTSCD) [41] and low-temperature supercritical drying (LTSCD) from $\mathrm{CO}_{2}$ [42].

HTSCD is carried out in three steps. Firstly, the aged gel is placed in an autoclave filled halfway with the same solvent held in the gel's pores. The vessel is then sealed and heated slowly past the solvent's critical temperature and pressure (i.e., most-used organic solvents have a relatively high $T_{\text {cr }}$ of $300-600 \mathrm{~K}$ with $P_{\text {cr }}$ of $30-80$ atm. Secondly, the fluid is isothermally depressurised. Finally, at ambient pressure, the autoclave is cooled to room temperature. In the case of silica aerogels, methanol is most frequently used as a solvent for HTSCD. At its critical point (i.e., $T_{\mathrm{cr}}=512.6 \mathrm{~K}, P_{\mathrm{cr}}=79.783 \mathrm{~atm}$ [45]), methanol can react with $\mathrm{OH}$ groups on the surface of the gel backbone to form $\mathrm{CH}_{3} \mathrm{O}$ groups, which make the silica aerogels partially hydrophobic and it is the reason why HTSCD silica aerogels are generally of higher quality. Furthermore, HTSCD has been found as the best way to minimise shrinkage of the gel. For each possible solvent, drying pressures are known for which shrinkage of the aerogels stays below 5\% [43].

The process of LTSCD is similar to that of HTSCD and is also carried out in three steps. Firstly, the aged gel is placed in an autoclave, but now filled with the safer, non-flammable liquid $\mathrm{CO}_{2}$ at $4-10{ }^{\circ} \mathrm{C}$ until 100 bar to replace the solvent in the pores of the gel. When all solvents are replaced, the autoclave is heated to $313 \mathrm{~K}$ while maintaining the pressure at 100 bars. Secondly, the fluid is isothermally depressurised. Finally, at ambient pressure, the autoclave is cooled to room temperature. In addition, aerogels dried by LTSCD show shrinkage but compared to HTSCD, it is not caused by SCD process but by the replacement of the original solvent with liquid $\mathrm{CO}_{2}$ [43].

\subsubsection{Ambient pressure drying}


APD is of most interest to lower the costs compared to the expensive drying processes of HTSCD or LTSCD. APD is generally carried out in two steps. Firstly, silylation of all OH groups must take place to prevent adsorption of water resulting from the formation of hydrophobic aerogels. This is carried out by replacing the present solvent with a water-free solvent and a silylating agent (e.g., hexamethyldisilazane (HMDS)), resulting in the replacement of $\mathrm{H}$ from $\mathrm{OH}$ groups by an alkyl such as $\mathrm{CH}_{3}$. Secondly, drying is carried out by ambient pressure evaporation [42] and consists of three steps. After a warming period, the first drying period occurs where the volume loss of the gel balances those of the evaporated liquid as free water moves continuously to the external surface by capillary forces. In the second drying period or falling rate period, diffusive vapour transport will dominate and allow liquid to escape slowly to the exterior.

\section{Aerogel-based adsorbents}

\subsection{Aerogels in carbon dioxide capture}

The application of aerogels as an amine-supported sorbent to capture $\mathrm{CO}_{2}$ gas is quite promising due to their numerous advantages such as low density, high porosity, and high surface area. In addition, due the versatility of the sol-gel process, the microstructural pattern, as well as composition and surface chemistry of aerogels could be deliberately tailored for $\mathrm{CO}_{2}$ capturing purposes $[16,44]$. For this reason, recent investigations have focused on using aerogels as new $\mathrm{CO}_{2}$ capture materials by manipulating the chemophysical properties of different aerogels. In this regard, amine-modified $\mathrm{SiO}_{2}$ aerogels using 3aminopropyltriethoxysilane (APTES) [27], polyethyleneimine, and tetraethylenepentamine [45] have been explored with $\mathrm{CO}_{2}$ adsorption capacity of $6.97 \mathrm{mmol} \mathrm{g} \cdot\left(\right.$ in $10 \% \mathrm{CO}_{2}+\mathrm{N}_{2}$ ) and $7.9 \mathrm{mmol} \mathrm{g}_{-1}$ under simulated flue gas conditions (in prehumidified $10 \% \mathrm{CO}_{2}$ ), respectively [46]. Recently, 3-aminopropyltrimethoxysilane (APTMS) and polyethyleneimine [47] modified particulate silica aerogels derived from water glass have been developed by Ananthakumar and co-workers [48]. They studied the effect of amine loading and its efficiency in the absorption of $\mathrm{CO}_{2}$ gas and correlated the sorption capacity with respect to amine functionality/contents and pore structure of the adsorbent. Scientists at Aspen Aerogels [49] developed amino-functionalised aerogels out of superhydrophobic methyltrimethoxysilane (MTMS) precursor co-condensed with amino-alkyl trialkoxysilane co-precursors. They obtained a capacity of approximately $1.8 \mathrm{mmol} \mathrm{g}^{-1}$ under humid $\mathrm{CO}_{2}$ $(15 \%)$ at $40{ }^{\circ} \mathrm{C}$ with excellent cyclic stability over 2,000 cycles.

\subsection{Aerogels in removal of heavy metal ions}

Adsorption experiments with heavy metal ions are also conducted with aerogel-based materials as the relevant number of publications in the field is increasing. Heavy metals such as chromium $(\mathrm{Cr})$, copper $(\mathrm{Cu})$, lead $(\mathrm{Pb})$, mercury $(\mathrm{Hg})$, manganese $(\mathrm{Mn})$, cadmium $(\mathrm{Cd})$, nickel $(\mathrm{Ni})$, zinc $(\mathrm{Zn})$, and iron $(\mathrm{Fe})$ are known to be highly toxic and nonbiodegradable for ecological environment and human being health, which can cause severe damages to liver, kidney, disorders in bone, and cancer.

One of the efficient ways to capture these heavy metal ions is to chelate them with some functional groups containing $\mathrm{N}, \mathrm{O}, \mathrm{S}$, and $\mathrm{P}$ as donor atoms, which are capable of forming a coordination complex with different metal ions [50]. The major advantage of solgel reaction is the possibility of tailoring the surface chemistry with various chelating groups by using a series of organically-modified silica precursors [51]. In this regard, 
amine-modified silica xerogels were prepared by Kłonkowski et al. [52] by anchoring (3aminopropyl)trimethoxysilane and (3-(2-aminoethyl)aminopropyl) trimethoxysilane to the xerogel surface and interior sections with high feasibility of chemisorption of $\mathrm{Cu}$ ions through complexation. Also, silica gels modified with several aliphatic mono-, di-, tri-, and tetramine-based functionalities possessed an interesting capturing efficiency towards several heavy metal ions [53]. Anchoring dithiocarbamate moieties using sialylation chemistry was also reported for capturing trace amounts of metal ions [50].

Motahari et al. (2016) developed amino-modified RF aerogels to capture $\mathrm{P}, \mathrm{Hg}$, and $\mathrm{Cd}$ ions from aqueous solution [54]. They studied the variation of several sorption parameters such as temperature, $\mathrm{pH}$, contact time, sorbent concentration, and initial metal concentration on the sorption capacity variations. They found that the sorption process was totally a $\mathrm{pH}$ dependent procedure and the maximum sorption capacity of $156.25,158.73$, and $151.52 \mathrm{mg} \mathrm{g}^{-1}$ could be obtained following an endothermic process in $1 \mathrm{~h}$ and at $\mathrm{pH} 6,6$, and 5 for metal ions of $\mathrm{Pb}, \mathrm{Hg}$, and $\mathrm{Cd}$, respectively. Also, the adsorption data could perfectly be fitted to both the Langmuir and Freundlich models. They ascribed the formation of a complex between heavy metal ions and surface amine groups as an adsorption mechanism of ions on the surface of aerogels.

\subsection{Aerogel in adsorption of water vapour}

The need to improve the performance of separation systems and heat transformation devices promotes the development of more effective adsorbents of water vapour. Twocomponent $\mathrm{SiO}-\mathrm{CaCl}_{2}$ or $\mathrm{SiO}-\mathrm{LiBr}$ aerogels exhibited adsorption capacities of water vapour in the range of $0.9-1.1 \mathrm{~kg} \mathrm{H}_{2} \mathrm{O} \mathrm{kg}^{-1}$ adsorbent, which is markedly higher than that of any known solid inorganic adsorbent [35]. Upgrading of silica gels by incorporating $\mathrm{CaCl}_{2}$ by means of impregnation recently improved adsorption capacities to $70 \mathrm{wt}$. \%. The authors found that the materials similar in composition to $\mathrm{SiO}$-hygroscopic substance $(\mathrm{SH})$ prepared by the sol-gel route appeared to be superior both in adsorptive capacity and overall performance. Adsorption properties of the materials remained stable during at least 60 adsorption-desorption cycles. 


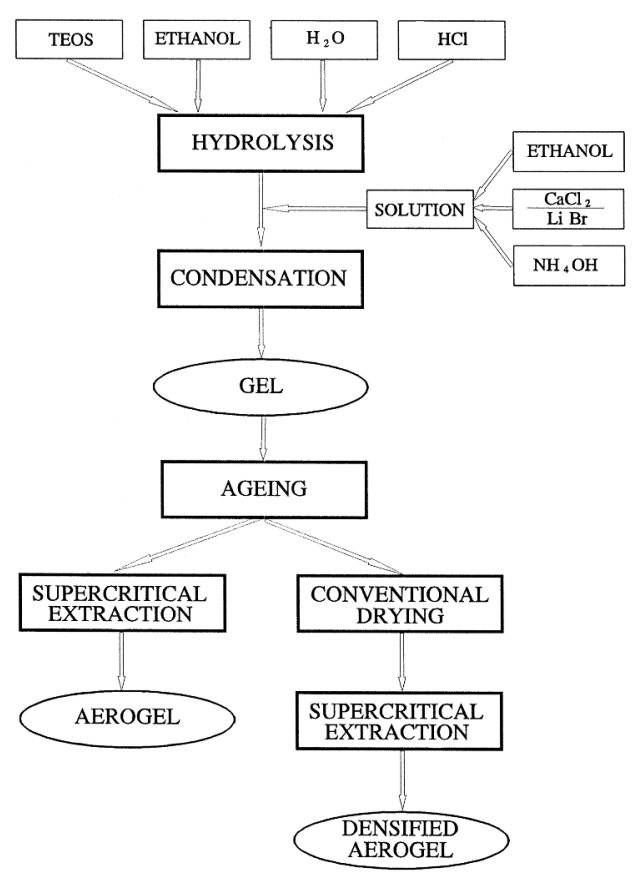

Figure. 1. Schematic representation for the preparation of two-component $\mathrm{SiO}-\mathrm{CaCl}_{2}$ or $\mathrm{SiO}-\mathrm{LiBr}$ aerogels [35].

The aerogels were prepared using the procedures outlined in Figure 1. Alcogel samples were synthesised to achieve the target of $30 \mathrm{wt}$. \% content of $\mathrm{CaCl}_{2}$ or $\mathrm{LiBr}$. In the first stage, TEOS was hydrolysed under reflux with the water content determined to match the total hydrolysis ratio and the water introduced into the system in the second stage with the solution of $\mathrm{CaCl}_{2}$ or $\mathrm{LiBr}$ was accounted. In the second stage, the solution was mixed with the remaining $\mathrm{EtOH}, \mathrm{NH}_{4} \mathrm{OH}$, and 35 wt. \% aqueous solution of HS. Alcogels were aged for six days and then dried in supercritical $\mathrm{CO}_{2}(15 \mathrm{MPa})$ at $45{ }^{\circ} \mathrm{C}$ to obtain bicomponent SiO-HS aerogels in a covered vessel at ambient conditions until about 50 wt. $\%$ of EtOH was removed, and then in supercritical $\mathrm{CO}_{2}$ to obtain densified aerogels. Interestingly, the densified aerogels appeared to exhibit more compact morphology but the pores are still quite open, as can be inferred from the values of mesopore volume detected by the nitrogen adsorption test.

\subsection{Aerogels in removal of volatile organic compounds}

Adsorption is the most common way to remove vapour of volatile compounds from the atmosphere. Aerogels can easily meet the adsorption requirements and their application in the removal of the aforementioned toxic pollutants is very promising [36]. Activated carbon aerogel is reported as a good candidate to capture toluene vapour with an excellent feasibility of regeneration $[36,55]$. The maximum toluene adsorption capacity of carbon aerogels with controlled pore size was $1,180 \mathrm{mg} \mathrm{g}-$ with the possibility of complete desorption at $400{ }^{\circ} \mathrm{C}$ [56]. Silica aerogel was also indicated as a promising sorbent to adsorb benzene vapour with saturation adsorption capacity of $3,000 \mathrm{mg} \mathrm{g}^{-1}$ whereas it was reported that the adsorption capacity of activated carbon powder was $500 \mathrm{mg} \mathrm{g}^{-1}$ [57]. Silica aerogels modified with methyltrimethoxysilane (MTMS) and trimethylethoxysilane (TMES) were also able to remove vapour from waste gas streams [58]. Surface hydrophobisation has been conducted to increase the moisture resistance of aerogels and 
improve the reusability. The results demonstrated that although the surface-modified silica aerogels demonstrated good performance towards capturing vapour with over 14 repeated adsorption-desorption regeneration cycles, the adsorption was less significant compared to the hydrophilic pristine silica aerogels due to the reduction in the BET surface area and micropores upon surface modification.

\section{Conclusion}

Aerogel-based materials have environmentally-friendly renewability, biocompatibility, and also excellent properties such as low density, high porosity, and high specific surface area. These remarkable properties combined with modifiable surface chemistry from the versatility of their wet synthesis approach have made these materials as a suitable candidate for environmental protection purposes. In most studies, aerogels have been found to be excellent sorbent materials with high sorption capacity towards the target components. The regeneration capability of aerogels during the process is also promising from economically and large-scale production point of view. Nevertheless, more works must be done to address several issues regarding the cost of production as the production of aerogels is quite lengthy and pristine aerogels are very fragile. The mechanical strength of aerogels should be improved, especially the compressive strength towards the preparation of highly flexible monoliths in which the aerogel is deliberately compressed.

This work was financially supported by Universiti Teknologi Malaysia and Malaysia-Thailand Joint Association (MTJA) programme (Vot. No. 4C116).

\section{References}

1. Fricke, J. and T. Tillotson, Aerogels: production, characterization, and applications. Thin Solid Films, 1997. 297(1): p. 212-223.

2. Akimov, Y.K., Fields of Application of Aerogels (Review). Instruments and Experimental Techniques, 2003. 46(3): p. 287-299.

3. Yin, W. and D.A. Rubenstein, Biomedical Applications of Aerogels, in Aerogels Handbook, M.A. Aegerter, N. Leventis, and M.M. Koebel, Editors. 2011, Springer New York: New York, NY. p. 683-694.

4. Gurav, J.L., et al., Silica Aerogel: Synthesis and Applications. Journal of Nanomaterials, 2010. 2010: p. 11.

5. Nicola, H. and S. Ulrich, Aerogels-Airy Materials: Chemistry, Structure, and Properties. Angewandte Chemie International Edition, 1998. 37(1-2): p. 22-45.

6. Mi, X., et al., Preparation of graphene oxide aerogel and its adsorption for $\mathrm{Cu}_{2}+$ ions. Carbon, 2012. 50(13): p. 4856-4864.

7. Sui, Z., et al., Green synthesis of carbon nanotube-graphene hybrid aerogels and their use as versatile agents for water purification. Journal of Materials Chemistry, 2012. 22(18): p. 8767-8771.

8. Guo, F., et al., Highly stretchable carbon aerogels. Nature Communications, 2018. 9(1): p. 881 . 
9. Chen, C., et al., Compressive, ultralight and fire-resistant lignin-modified graphene aerogels as recyclable absorbents for oil and organic solvents. Chemical Engineering Journal, 2018. 350: p. 173-180.

10. Ge, X., et al., High-strength and morphology-controlled aerogel based on carboxymethyl cellulose and graphene oxide. Carbohydrate Polymers, 2018. 197: p. 277-283.

11. García-González, C.A., et al., Polysaccharide-based aerogel microspheres for oral drug delivery. Carbohydrate Polymers, 2015. 117: p. 797-806.

12. Rajendar, R.M., et al., Silk fibroin aerogels: potential scaffolds for tissue engineering applications. Biomedical Materials, 2015. 10(3): p. 035002.

13. Rudaz, C., et al., Aeropectin: Fully Biomass-Based Mechanically Strong and Thermal Superinsulating Aerogel. Biomacromolecules, 2014. 15(6): p. 2188-2195.

14. Veronovski, A., et al., Characterisation of biodegradable pectin aerogels and their potential use as drug carriers. Carbohydrate Polymers, 2014. 113: p. 272278.

15. Barnyakov, A.Y., et al., Threshold aerogel Cherenkov counters of the KEDR detector. Journal of Instrumentation, 2014. 9(09): p. C09005.

16. Pierre, A.C. and G.M. Pajonk, Chemistry of Aerogels and Their Applications. Chemical Reviews, 2002. 102(11): p. 4243-4266.

17. Tonguc, B.T. and S. Citci, Aerogel Efficiencies of Threshold Cherenkov Counters. Arabian Journal for Science and Engineering, 2014. 39(7): p. 5739-5743.

18. Sabri, F., et al., Effect of Aerogel Particle Concentration on Mechanical Behavior of Impregnated RTV 655 Compound Material for Aerospace Applications. Advances in Materials Science and Engineering, 2014. 2014: p. 10.

19. Randall, J.P., M.A.B. Meador, and S.C. Jana, Tailoring Mechanical Properties of Aerogels for Aerospace Applications. ACS Applied Materials \& Interfaces, 2011. 3(3): p. 613-626.

20. Wei, G., X. Zhang, and F. Yu, Effective thermal conductivity analysis of xonotliteaerogel composite insulation material. Journal of Thermal Science, 2009. 18(2): p. 142-149.

21. Júlio, M.d.F. and L.M. Ilharco, Superhydrophobic hybrid aerogel powders from waterglass with distinctive applications. Microporous and Mesoporous Materials, 2014. 199: p. 29-39.

22. Habib Ullah, M., W.N.L. Mahadi, and T.A. Latef, Aerogel Poly(butylene succinate) Biomaterial Substrate for RF and Microwave Applications. Scientific Reports, 2015. 5: p. 12868.

23. Veres, P., et al., Hybrid aerogel preparations as drug delivery matrices for low water-solubility drugs. International Journal of Pharmaceutics, 2015. 496(2): p. 360-370.

24. Mikkonen, K.S., et al., Prospects of polysaccharide aerogels as modern advanced food materials. Trends in Food Science \& Technology, 2013. 34(2): p. 124-136.

25. Power, M., et al., Aerogels as biosensors: viral particle detection by bacteria immobilized on large pore aerogel. Journal of Non-Crystalline Solids, 2001. 285(1): p. 303-308.

26. Mazhar, U.-I., et al., Bacterial cellulose composites: Synthetic strategies and multiple applications in bio-medical and electro-conductive fields. Biotechnology Journal, 2015. 10(12): p. 1847-1861.

27. Cui, S., et al., Mesoporous amine-modified $\mathrm{SiO}_{2}$ aerogel: a potential $\mathrm{CO}_{2}$ sorbent. Energy \& Environmental Science, 2011. 4(6): p. 2070-2074. 
28. Baetens, R., B.P. Jelle, and A. Gustavsen, Aerogel insulation for building applications: A state-of-the-art review. Energy and Buildings, 2011. 43(4): p. 761769.

29. Shaid, A., M. Fergusson, and L. Wang, Thermophysiological comfort analysis of aerogel nanoparticle incorporated fabric for fire fighter's protective clothing. Chemical and materials engineering, 2014. 2(2): p. 37-43.

30. Welsch, F., M. Nemec, and W. Lawrence, Two-generation reproductive toxicity study of resorcinol administered via drinking water to Crl: $C D$ (SD) rats. International journal of toxicology, 2008. 27(1): p. 43-57.

31. Welsch, F., Routes and Modes of Administration of Resorcinol and Their Relationship to Potential Manifestations of Thyroid Gland Toxicity in Animals and Man. International Journal of Toxicology, 2008. 27(1): p. 59-63.

32. Rigacci, A., et al., Aerogels: a fascinating class of materials with a wide potential of application fields. 2017. 84(3): p. 375-376.

33. Wang, X. and S.C. Jana, Synergistic Hybrid Organic-Inorganic Aerogels. ACS Applied Materials \& Interfaces, 2013. 5(13): p. 6423-6429.

34. Soleimani Dorcheh, A. and M.H. Abbasi, Silica aerogel; synthesis, properties and characterization. Journal of Materials Processing Technology, 2008. 199(1): p. 10-26.

35. Mrowiec-Białoń, J., et al., Two-component aerogel adsorbents of water vapour. Journal of Non-Crystalline Solids, 1998. 225: p. 184-187.

36. Fairén-Jiménez, D., F. Carrasco-Marín, and C. Moreno-Castilla, Adsorption of benzene, toluene, and xylenes on monolithic carbon aerogels from dry air flows. Langmuir, 2007. 23(20): p. 10095-10101.

37. Hüsing, N. and U. Schubert, Aerogels-Airy Materials: Chemistry, Structure, and Properties. 1998. 37(1-2): p. 22-45.

38. Hæreid, S., E. Nilsen, and M.-A. Einarsrud, Properties of silica gels aged in TEOS. Journal of Non-Crystalline Solids, 1996. 204(3): p. 228-234.

39. Smitha, S., et al., Effect of aging time and concentration of aging solution on the porosity characteristics of subcritically dried silica aerogels. Microporous and Mesoporous Materials, 2006. 91(1): p. 286-292.

40. Maleki, H., et al., Synthesis and biomedical applications of aerogels: Possibilities and challenges. Advances in Colloid and Interface Science, 2016. 236: p. 1-27.

41. Kistler, S.S., Coherent Expanded-Aerogels. The Journal of Physical Chemistry, 1931. 36(1): p. 52-64.

42. Tewari, P.H., A.J. Hunt, and K.D. Lofftus, Ambient-temperature supercritical drying of transparent silica aerogels. Materials Letters, 1985. 3(9): p. 363-367.

43. Kirkbir, F., et al., Drying of aerogels in different solvents between atmospheric and supercritical pressures. Journal of Non-Crystalline Solids, 1998. 225: p. 1418.

44. Rechberger, F., G. Ilari, and M. Niederberger, Assembly of antimony doped tin oxide nanocrystals into conducting macroscopic aerogel monoliths. Chemical Communications, 2014. 50(86): p. 13138-13141.

45. Qi, G., et al., High efficiency nanocomposite sorbents for $\mathrm{CO}_{2}$ capture based on amine-functionalized mesoporous capsules. Energy \& Environmental Science, 2011. 4(2): p. 444-452.

46. Linneen, N., R. Pfeffer, and Y.S. Lin, $\mathrm{CO}_{2}$ capture using particulate silica aerogel immobilized with tetraethylenepentamine. Microporous and Mesoporous Materials, 2013. 176: p. 123-131.

47. Zhang, S., et al., Irradiation-induced grafting of acrylonitrile onto activated carbon fiber. Polymers for Advanced Technologies, 2009. 20(12): p. 1168-1173. 
48. Minju, N., et al., Amine impregnated porous silica gel sorbents synthesized from water-glass precursors for $\mathrm{CO}_{2}$ capturing. Chemical Engineering Journal, 2015. 269: p. 335-342.

49. Redouane, B., et al., Superhydrophobic amine functionalized aerogels as sorbents for CO2 capture. Greenhouse Gases: Science and Technology, 2013. 3(1): p. 3039.

50. Leyden, D.E. and G.H. Luttrell, Preconcentration of trace metals using chelating groups immobilized via silylation. Analytical Chemistry, 1975. 47(9): p. 16121617.

51. Soliman, E.M., Synthesis and Metal Collecting Properties of Mono, Di, Tri and Tetramine Based on Silica Gel Matrix. Analytical Letters, 1997. 30(9): p. 17391751.

52. Kłonkowski, A.M., et al., The Coordination State of Copper(II) Complexes Anchored and Grafted onto the Surface of Organically Modified Silicates. Langmuir, 1999. 15(18): p. 5814-5819.

53. Soliman, E., Synthesis and Metal Collecting Properties of Mono, Di, Tri and Tetramine Based on Silica Gel Matrix. Vol. 30. 1997. 1739-1751.

54. Motahari, S., M. Nodeh, and K. Maghsoudi, Absorption of heavy metals using resorcinol formaldehyde aerogel modified with amine groups. Desalination and Water Treatment, 2016. 57(36): p. 16886-16897.

55. Dingcai, W., S. Zhuoqi, and F. Ruowen, Structure and adsorption properties of activated carbon aerogels. Journal of Applied Polymer Science, 2006. 99(5): p. 2263-2267.

56. Maldonado-Hódar, F.J., et al., Reversible toluene adsorption on monolithic carbon aerogels. Journal of Hazardous Materials, 2007. 148(3): p. 548-552.

57. Zhang, Z., et al. Benzene adsorption properties of silica aerogel-fiber composites. in 2008 2nd IEEE International Nanoelectronics Conference. 2008.

58. S Standeker, S., Z. Novak, and Ž. Knez, Removal of BTEX vapours from waste gas streams using silica aerogels of different hydrophobicity. Journal of Hazardous Materials, 2009. 165(1): p. 1114-1118. 